\title{
Análisis del uso del antiguo material gimnástico y de atletismo de la Ley General de Educación en las clases de Educación Física actual \\ Analysis of the use of the old gymnastic and athletics material of the General Law on Education in present physical education classes \\ JoséEugenio Rodríguez-Fernández, CristianAbelairas-Gómez, Lucía Peixoto-Pino \\ Universidad de Santiago de Compostela (España)
}

Resumen. La Educación Física (EF) en la Ley General de Educación (LGE) de 1970 se alimentaba de los principios generales de este sistema educativo: un modelo cerrado, con una evaluación basada en el producto final, observable y con el planteamiento de objetivos terminales. Dentro de los objetivos generales de la materia de EF estaba «el desarrollo de la capacidad físico-deportiva», diseñando sesiones, actividades y empleando materiales que ayudaran a conseguir ese propósito. El objetivo de esta investigación fue describir el tipo de material gimnástico y de atletismo característico de la LGE que disponían los centros educativos en los que se imparte Educación Primaria en Galicia y analizar su uso en las clases de EF en la actualidad. Participaron un total de 226 centros (34,61\% del total de la población objeto de estudio) a través del docente especialista en EF, quien respondió a un cuestionario sobre el material gimnástico y de atletismo de su centro. Los resultados nos muestran una alta disponibilidad de uso de material gimnástico y de atletismo por su presencia en las instalaciones del centro, sin embargo, los docentes hacen un escaso uso de este material en sus sesiones, por tratarse de material anticuado, difícil de manejar y que, en casos, puede resultar peligroso su uso y manipulación por el alumnado. Además de potenciar su uso específico, los docentes deberían buscar un uso alternativo a este tipo de material, ampliando y diversificando el uso de diferentes materiales didácticos para mejorar el proceso de enseñanza-aprendizaje en el ámbito de la EF.

Palabras clave. Ley General de Educación, Educación Física, Educación Primaria, material didáctico, recursos.

\begin{abstract}
In the 1970 General Law on Education (LGE), Physical Education was supplied with the general principles of this educational system: a closed model, which had an observable evaluation based on the final product and focused on terminal objectives. Within the general objectives of the Physical Education subject, it was found «the development of the physical-sporting capacity», designing lessons, activities and using materials that help to achieve that purpose. The objective of this research was to describe the type of gymnastic and athletic materials, characteristic of this law, available in the Galician educational centers where Primary Education was offered, and to analyze their use in Physical Education classes today. A total of 226 educational centers participated (34.61\% of the total population under study) through the specialist teacher in Physical Education, who have answered a questionnaire about the aforementioned materials from their centers. The results show us a high availability of its use due to its presence in the facilities of the center. However, teachers make little use of these materials in their lessons, as it is outdated, difficult to handle, and which, in some cases, can be dangerous and manipulated by students. In addition to enhancing their specific use, teachers should search for an alternative use to this type of material, increasing ad diversifying the use of different didactic materials to improve the teaching-learning process in the field of Physical Education.. Keywords. General Education Law, physical education, primary education, teaching material, resources.
\end{abstract}

\section{Introducción}

Los materiales para el área de Educación Física son definidos como el conjunto de elementos útiles que el profesor utiliza como soporte y que complementan su acción docente (Díaz-Lucea, 1996).

En 1970 la Ley General de Educación y Financiamiento de la Reforma Educativa, conocida por el acrónimo LGE, ordenó un nuevo sistema educativo, contemplando a la Educación Física como una materia cursada desde la EGB hasta el Bachillerato. Las dotaciones de equipamiento tradicionalmente tuvieron una orientación gimnástica, atlética e higienista, basada en material convencional, algunos de los cuales siguen siendo comunes como los bancos suecos, las colchonetas o las espalderas (Tabernero \& Márquez, 2003). Estos materiales son los que Blández (1995) define como específicos del área de Educación Física clasificándolos en material de gimnasio, de patio, de deporte o de psicomotricidad.

El tipo de materiales y equipamientos desde entonces hasta la década de los 90 fueron principalmente los que poseían un carácter físico-deportivo (Molina, Devís, \& Peiró, 2008) y gimnástico. Es en este momento cuando el material no convencional, de reciclaje o autoconstruido empezó a cobrar especial importancia (MéndezGiménez, Fernández-Río, \& Méndez-Alonso, 2012), bien como reacción a la relación ideológica con los materiales convencionales de Educación Física (tales como las espalderas, potro, plinto, trampolín o colchonetas) o bien como una nueva expresión didáctica.

Bajo esta nueva concepción de la Educación Física, en la que los materiales gimnásticos y atléticos, en muchos casos, fueron postrados a los almacenes de los centros, subyacía una nueva concepción de la Educación Física basada en estrategias menos directivas, alejadas de la gimnástica por asociación ideológica a la educación franquista y con el inestimable apoyo de editoriales y empresas que ponían en el mercado nuevos equipamientos. La aparición continua de materiales novedosos para el área de Educación Física es un hecho constatado en las últimas décadas (Tabernero \& Márquez, 2003).

En este sentido Tabernero y Márquez (2003) en el cambio de milenio, observaron dos posicionamientos opuestos sobre el uso de material por parte de los profesores de Educación Física. Por un lado, estaban los que entendían que todo material era poco (denominado por los autores materialismo pedagógico) y en el polo opuesto aquellos que entendían que no era necesario demasiado material para la práctica docente. En este nuevo siglo, a nivel escolar se aprecia un dualismo ideológico en muchos profesores de cómo plantear la Educación Física, también relacionada con aquel grupo de maestros partidarios de actividades físicas con finalidades higienistas o deportivas, y aquellos otros que promueven una Educación Física ciudadana, basada en la cooperacióny, en definitiva, con relativa oposición a las estructuras cerradas del deporte.

En el área de Educación Física, los materiales siempre han sido un recurso fundamental en la planificación docente y ligados a las actividades educativas (Díaz- Lucea, 1996). En los primeros años escolares el alumnado debería tener diversas oportunidades para el desarrollo funcional del movimento, basado en múltiples experiencias prácticas(Maeda \& Burt, 2003), habiendo evidencias de que, prácticas estructuradas ya desde la educación infantil, promuenven un mayor desarrollo motor (Teixeira-Costa, Barcala-Furelos, Abelairas-Gómez, \& Arufe-González, 2015). Para los niños, el equipamiento puede ofrecer diferentes propuestas y usos en relación con la creatividad de los maestros o la imaginación de los alumnos (Maeda \& Burt, 2003).

Los materiales gimnásticos se caracterizan per se por su durabilidad y también por su uso finalista. Fueron creados para algo concreto (por ejemplo, el potro). Una provisión limitada de equipamiento, bien en cantidad o bien en calidad, puede afectar negativamente al desarrollo del 
currículo de Educación Física (Méndez-Giménez et al., 2012); sin embargo, el coste de material no es un prerrequisito para la efectividad de los programas basados en la motricidad ya que, desde tiempos inmemoriales, el profesorado ha usado su creatividad e innovación para diseñar o reutilizar materiales que no supongan un coste económico (Maeda \& Burt, 2003).

A nivel global, la precariedad es un común denominador en los equipamientos y materiales en el área de Educación Física (FernádezRío, 2010; Maeda \& Burt, 2003), por tanto, el uso de materiales obsoletos es una realidad en la práctica docente (Méndez-Giménez et al., 2012). Las inadecuadas instalaciones y equipamientos para la enseñanza de la Educación Física a menudo se asocian a pocos recursos económicos ocurriendo esto en mayor o menor medida en todos los continentes (Hardman, 2008).

De este estudio se pudo constatar que en la mayoría de los países la provisión de materiales es mínima. La calidad de los equipamientos y materiales en el 26\% de Europa y 58\% en América Latina están por debajo de lo adecuado y la cantidad de elementos es limitada o insuficiente en un 41\% para Europa y en América Latina alcanza el 87\% de las encuestas. Los resultados de esta investigación chocan con los postulados de la Organización de las Naciones Unidas para la Educación, la Ciencia y la Cultura (UNESCO), que promueve la calidad en la enseñanza de la Educación Física con estrategias como la creación de entornos adecuados de aprendizaje y la mejora de equipamientos y materiales (United Nations Educational, Scientific and Cultural Organizations, 2015).

Diversos autores consideran el material de Educación Física fundamental en los procesos de enseñanza aprendizaje (Barcala-Furelos, Abelairas-Gómez, \& Gil-Madrona, 2016; Blández, 1995; Tabernero \& Márquez, 2003).

El carácter práctico de la Educación Física, la coexistencia de recursos en los centros educativos correspondientes a diferentes décadas y la falta de estudios recientes sobre la existencia y usabilidad de material convencional gimnástico y atlético, hace que este tópico vuelva a cobrar importancia para los educadores físicos. La utilización de material convencional de forma no convencional que ha definido Díaz-Lucea(1996) englobando las usanzas alternativas (diferentes a la finalidad que fueron construidos) o la emergente metodología basada en ambientes de aprendizaje (Barcala-Furelos et al., 2016) han provocado una redimensión de los espacios y equipamientos (clásicos y modernos).

Fernández-Río (2010) ordena en tres líneas (revisionista, autoconstrucción y ecológica) los recursos materiales en función de las posibilidades que nos ofrecen para una práctica renovada. La línea revisionista pretende dar nuevos usos a muchos recursos tradicionales (Fernádez-Río, 2010). Los materiales de Educación Física deben ser una fuentedeestímulos, se les presupone polivalencia, multifuncionalidad y además rentabilidad (Tabernero \& Márquez, 2003).

Por tanto, el objetivo de este estudio es conocer la existencia, grado de conservación y frecuencia de uso del material gimnástico y atlético tradicional por los maestros de Educación Física.

\section{Material y método}

\section{Diseño}

Optamos por un estudio mixto cualitativo-cuantitativo como estrategia metodológica (Johnson \& Onwegbuzie, 2004; Pereira, 2011; Ugalde \& Balbastre, 2013), encuadrado bajo un paradigma descriptivo (Amado, 2014; Lima \& Palhares, 2014). Se opta por este tipo de metodología para acercarnos a la realidad a estudiar (los centros educativos) y su contexto natural (Flick, 2015), caracterizando un fenómeno o situación concreta (uso de materiales en Educación Física) e indicando sus rasgos más peculiares o diferenciadores.

\section{Muestra}

En cuanto a centros de carácter público, la Comunidad Autónoma de Galicia dispone de 565 Centros de Educación Infantil y Primaria (CEIP), 23 Centros de Educación Primaria (CEP) Y 65 Centros Públi- cos Integrados (Xunta de Galicia, 2018). Un total de 653 centros educativos que disponen de instalaciones deportivas propias y que, además, cuentan con, al menos, un docente especialista en Educación Física. En esta investigación se invitó a participar a la totalidad de los centros.

Finalmente, participaron226 centros educativos (34,61\% de la población objeto de estudio), cuya información fue adquirida del docente especialista en Educación Física.

\section{Instrumento}

Se utilizó un cuestionario no validado como instrumento de recogida de datos, optando por el e-research o proceso de investigación en línea (Anderson \& Kanuka, 2002; Rodríguez-Gómez \& Valldeoriola, 2009) para su difusión y recogida de datos. Se trató de un cuestionario de 11 ítems, de carácter mixto (preguntas cerradas que dan opción al encuestado a razonar, matizar o ampliar su respuesta a través de la opción «otros» u «observaciones»). De estas 11 preguntas, 6 fueron de elecciónmúltiple,4deescala tipo Likerty1 deelecciónúnica(dicotómica). La primera de las preguntas hacía referencia al espacio disponible para la práctica deEducación Física del centro. Las ocho siguientes buscaban recopilar información sobre la existencia, estado de conservación, frecuencia de uso y razón por la cual no es usado el material de gimnasia (cuatro preguntas) y atletismo (cuatro preguntas). La décima pregunta interrogaba sobre el material de fabricación de y laúltima sobre el material empleado habitualmente en las sesiones de Educación Física (Anexo I).

\section{Procedimiento}

Se estableció la siguiente hoja de ruta para la toma de datos y su posterior tratamiento:

- Se procede con una revisión de la literatura científica y específica sobre los materiales didácticos en Educación Física. Drive

Se elabora un cuestionario a partir de un formulario en Google

- Localizados los centros públicos de Galicia en los que se imparte Educación Primaria, se envía por correo electrónico al centro educativo una breve presentación para la dirección del centro, una carta de presentación para el docente especialista en Educación Física y el cuestionario elaborado por los investigadores. No se tuvieron en cuenta los Centros Rurales Agrupados o «CRA» (28 en Galicia, 14 que sólo imparten Educación Infantil y 14 que imparten ambas etapas educativas) por no contar con un docente especialista en Educación Física ni instalaciones deportivas con el material didáctico objeto de investigación.

- El cuestionario estuvo operativo entre el 15/01/2018 y el 28/01/ 2018

- Los datos recogidos se almacenan en la propia plataforma de Drive, realizando este programa automáticamente el análisis estadístico (porcentajes), con los que se realiza posteriormente el tratamiento y análisis de datos.

\section{Resultados}

La estructuración de los resultados se expone con el siguiente guion: 1) espacios destinados a la Educación Física; 2) descripción del material gimnástico de los centros; 3) descripción del material de habilidades atléticas; y 4) material utilizado por los docentes en las sesiones de Educación Física.

Los centros educativos de Educación Primaria de Galicia disponen, sobre todo, de un pabellón polideportivo cubierto (59.7\%) o un gimnasio de interior (57.1\%). Es en estas dos instalaciones donde se almacena y se hace uso del material gimnástico mostrado en la figura 1. En porcentajes menores, un $29.6 \%$ de los centros disponen de una sala de psicomotricidad (destinada preferentemente a alumnado de Educación Infantil), un 18,1\% de una sala multiusos y, de forma complementaria, otros espacios que se usan para la práctica físico-deportiva de forma esporádica: pistas exteriores, patios cubiertos y descubiertos, zonas verdes y arboladas, entre otras.

En cuanto al material gimnástico disponible en los centros educati- 
vos analizados (figura 1), destaca la presencia de espalderas (91\%); en menor proporción, los plintos (68.6\%), potros (55.7\%) y minitramps (42.9\%); las cuerdas verticales para trepar (20.5\%) y el trampolín (19.5\%) están presentes en una cuarta parte de esos centros y, en porcentajes menores, las barras de equilibrio (16.7\%), camas elásticas (11\%), caballos (10\%) y escaleras horizontales (10\%). Materiales como barras (paralelas, fija o asimétricas), anillas o caballo con arcos están presentes tan sólo en el 1.26\% de los casos.

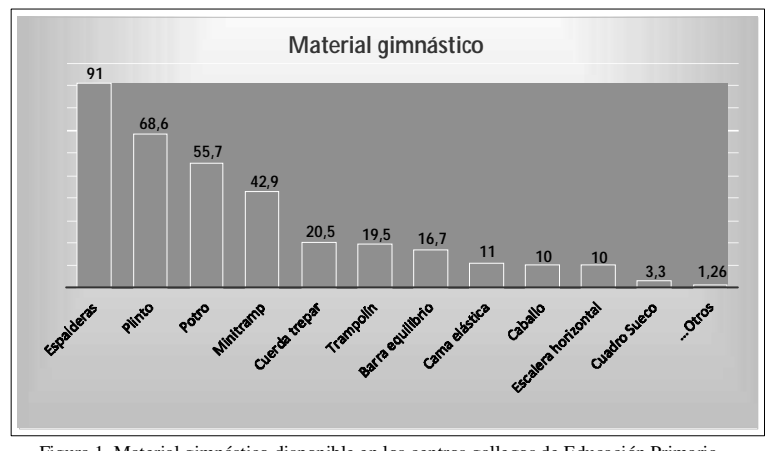

En la figura 2 se muestra el estado de conservación de este material gimnástico disponible en los centros y su frecuencia de uso en las clases de Educación Física. Por un lado, observamos que este material está en buen estado de conservación (51.9\%) y, en el 34\% de los casos, regular. Los extremos (excelente estado de conservación o muy malo) son los porcentajes menores, con $4.4 \%$ y $2.4 \%$ respectivamente.

Por otro lado, observamos también en esta figura 2 que la frecuencia de uso de este material gimnástico por parte de los docentes especialistas en Educación Física en sus clases es baja o muy baja. Así, se muestran porcentajes de uso de 39.1\% para el apartado de «algunas veces» $\mathrm{y}$ de $42.5 \%$ para el de «pocas veces». Asimismo, es preciso destacar que, dentro de este bajo uso del material gimnástico, el que más se utiliza son las espalderas, sin ser necesariamente para uso concreto de ejercicios gimnásticos. Otro material como el minitramp, la barra de equilibrios el plinto y las cuerdas de trepar también se usan ocasionalmente, siendo prácticamente nulo el uso del resto del material gimnástico.

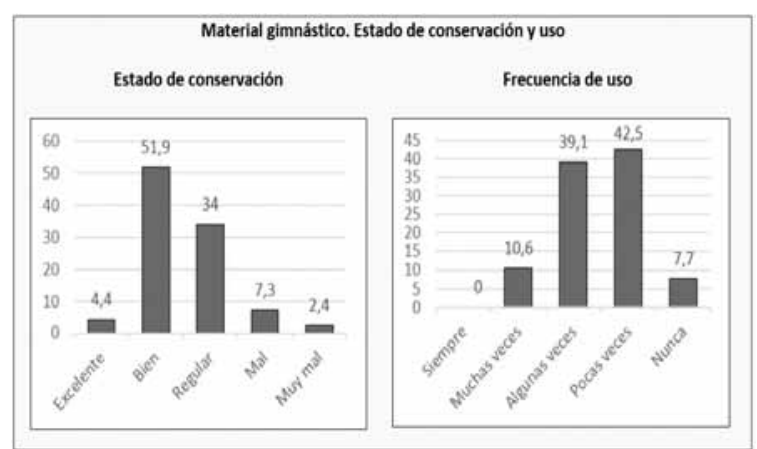

Figura 2. Estado de conservación y frecuencia de uso del material gimnástico.

A pesar de disponer de este material como recurso didáctico, muchos docentes no hacen uso de él. Los motivos se deben preferentemente a que creen que su uso puede ser peligroso para los participantes (58.6\%) y porque es complicado manejarlo, debido a su forma y excesivo peso (43.6\%). El 18\% opina que es un material que ha quedado anticuado, que actualmente hay otro tipo de material más moderno que suple las funciones de este material que ha quedado obsoleto; y el 3\% afirma desconocer o no dominar la actividad para la que fueron creados y/o diseñados estos elementos. Otros motivos expuestos por los propios docentes $(0.8 \%)$ se refieren a que los contenidos actuales del currículum no invitan precisamente al uso de este tipo de material, debido a su mal estado de conservación o porque se pueden conseguir los objetivos curriculares propuestos empleando otros materiales más atractivos, menos costosos, más manejables y menos peligrosos para los participantes debido a su uso.
En referencia al material para la práctica del atletismo disponible en los centros educativos (figura 3), destaca la presencia de tres elementos: el balón medicinal (82.3\%), la colchoneta quitamiedos (78.6\%) y las vallas (60.9\%). Con porcentajes sensiblemente inferiores, también disponen de los elementos para la práctica del lanzamiento de peso (40\%), lanzamiento de disco (36.4\%) y el saltómetro (33.6\%) para la práctica de salto de altura, junto con el quitamiedos especificado anteriormente. Los materiales menos presentes en los centros son la jabalina (16.4\%), el martillo (5.9\%) y la pértiga (1.4\%). Otros materiales nombrados por los docentes, con porcentajes testimoniales $(0.5 \%)$ corresponden a testigos, mini vallas o jabalinas de foam, entre otros.

En la figura 4 se muestra el estado de conservación del material de atletismo disponible en los centros y su frecuencia de uso en las clases de Educación Física. Se observa que este material está en buen estado de conservación (59\%) y, en el 30.4\% de los casos, regular. Un 5.1\% opina que el material del que dispone está en malas condiciones, porcentaje similar a los que creen que el material disponible en su centro está en excelentes condiciones. El porcentaje más bajo (0.5\%) son para los que muestran que este material está en muy malas condiciones.

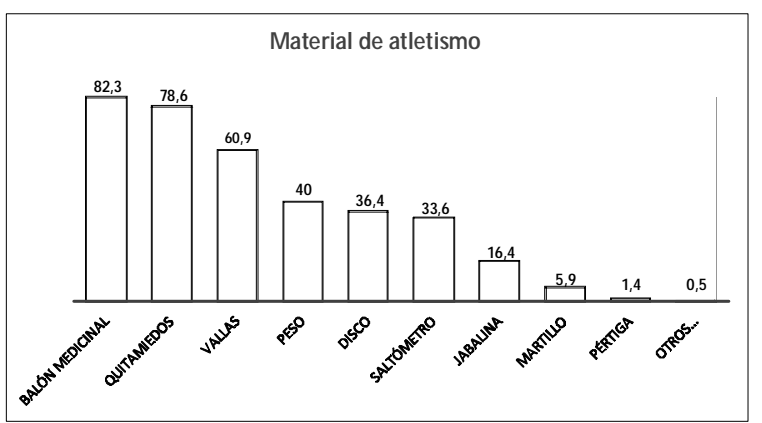

Figura 3. Material para la práctica del atletismo

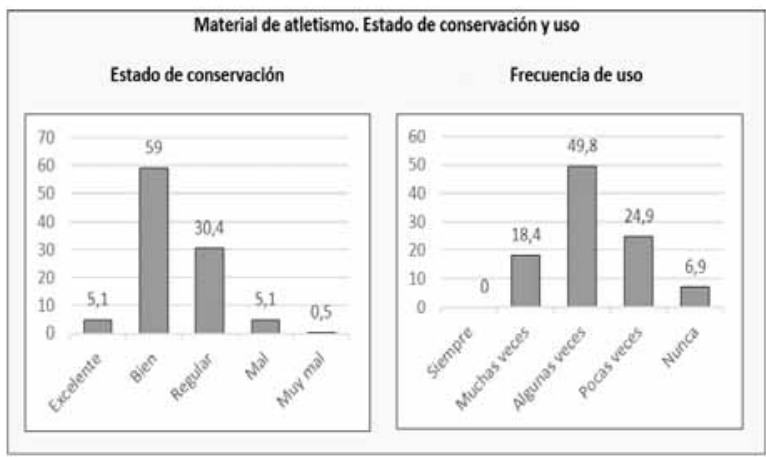

Figura 4. Estado de conservación y frecuencia de uso del material de atletismo.

Se puede apreciar también en esta figura 4 que la frecuencia de uso de este material gimnástico por parte de los docentes especialistas en Educación Física en sus clases es baja, mostrando porcentajes de uso de $49.8 \%$ para el apartado de «algunas veces» y de $24.9 \%$ para el de «pocas veces»; tan sólo el $18.4 \%$ afirma utilizarlo muchas veces y, ninguno, de forma habitual en sus clases. Asimismo, es preciso destacar que, dentro de este bajo uso del material de atletismo, el que más se utiliza son las vallas, la colchoneta quitamiedos y el balón medicinal, pero, curiosamente, no para la práctica específica del atletismo, sino que hacen un uso polivalente de este material para contenidos diferentes al de la práctica de atletismo (juegos, condición física o multideporte).

Observamos en la figura 4 que un 6,9\% de los docentes, a pesar de disponer de este material, no lo utiliza nunca o pocas veces (24.9\%). Los motivos se deben preferentemente a que utilizan otro tipo de materiales más actuales, más atractivos y fabricados con materiales más blandos y acolchados con los que consiguen los mismos objetivos que utilizando este material más anticuado (33\%), que incluso su uso puede suponer un cierto peligro para los alumnos (32\%) (por su peso o tipo de material empleado en su fabricación, como es el caso de la jabalina, el 
martillo, el peso o las vallas de madera). De hecho, afirman que la mayoría del material del que disponen es tradicional (63.3\%) (con materiales de fabricación basados en madera, hierro o aluminio), frente al $36.7 \%$ que responde que el material del que disponen es moderno, fabricado con materiales plásticos de baja densidad y predominando las texturas blandas y acolchados.

Un 20.6\% destaca también que su utilización y manejo es complicado, prefiriendo emplear otro tipo de materiales o, incluso, no realizar estetipo demodalidades deportivas con el alumnado. Deforma anecdótica (1\%) nos encontramos con razones como que su uso estropearía el pavimento de la instalación del centro, que se trata de un material demasiado específico para el alumnado deEducación Primaria(alumnado muy joven) o que, directamente, utilizan otro tipo de material más polivalente y adaptado a las características del alumnado.

En la figura 5 se muestra el tipo de material que los docentes emplean habitualmente en sus sesiones de Educación Física con el alumnado. Prácticamente, la mayoría (92\%) hace uso del material convencional, o sea, aquel diseñado específicamente para la práctica de actividad físico-deportiva; el 53.8\% emplea material convencional con un uso más polivalente, que le permita adaptarse a diferentes circunstancias (tipo de actividad deportiva, edad del alumnado, lugar de práctica... ) y, un 49.8\%, emplea material no convencional (aquel que no ha sido tradicionalmente utilizado en Educación Física, como puede ser el material autoconstruido, material reciclado, etc...).

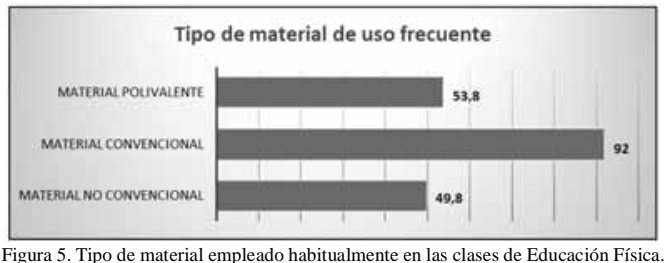

\section{Discusión}

El uso de material en la Educación Física es considerado un factor fundamental del proceso de enseñanza-aprendizaje (Barcala-Furelos et al, 2016) además de un importante elemento de innovación, junto al uso de los diferentes espacios, que ha permitido la evolución de la Educación Física en los últimos años (López-Pastor \& Gea-Fernández, 2010).

La enseñanza de las habilidades gimnásticas era considerada fundamental para el desarrollo del individuo (Buèar-Pajek, Èuk, Kovaè, \& Jakše, 2010). Por este motivo, los centros educativos fueron dotados de material específico para mencionada praxis. Estas enseñanzas no se han visto favorecidas en el sistema educativo en las últimas décadas (ÁvalosRamos, Martínez-Ruiz, \& Merma-Molina, 2015), algo que se refleja en los resultados de nuestro estudio al mostrar que más de la mitad del profesorado encuestado no hace uso del material gimnástico para sus sesiones o que su uso es poco frecuente. El mal estado en el que se encuentra el material gimnástico podría ser una razón para el descenso de la enseñanza de estos contenidos tan específicos, sin embargo, en nuestros resultados observamos que solamente un $10 \%$ del material se consideraba en mal o muy mal estado.

La carencia de formación universitaria del profesorado en habilidades gimnásticas (Ávalos-Ramos et al., 2015) podría ser una razón que afectara negativamente a su autopercepción, reduciéndose la enseñanza de estos contenidos (Cenizo-Benjumea, Seiva-Henares, \& FernándezTruán, 2017)y, por consiguiente, el uso del material destinado para ello. De hecho, casi dos terceras partes de los encuestados afirmaron no hacer uso del material por considerarlo peligroso.

En el caso del material de atletismo, encontramos resultados similares a los hallados respecto al material gimnástico. Aunque el estado de conservación es relativamente bueno, su uso queda relegado a «algunas veces», «pocas veces» 0 «nunca» en más de tres cuartas partes de los docentes encuestados. Este hecho podría ser explicado también porque, siendo considerado el atletismo uno de los deportes rey en la enseñanza de la Educación Física, no es estrictamente necesario el uso de espacios y materiales específicos para impartir este contenido (DelgadoFernández, Valero-Valenzuela, \& Conde-Caveda, 2003). En consonancia con esta línea argumental, una quinta parte delos docentes encuestados destacaron la complejidad de uso del material específico de atletismo, apuntando su preferencia por el uso de otro tipo de materiales para la enseñanza de las habilidades atléticas.

Al mismo tiempo que evolucionaron las metodologías de enseñanza de la Educación Física en los diferentes niveles del sistema educativo, también lo hicieron los materiales y las diferentes perspectivas sobre su uso. La aparición de nuevos materiales polivalentes, así como el uso de materiales alternativos no construidos con objetivos puramente enmarcados en el ámbito de la Educación Física, hizo que materiales con gran especificidad como los destinados a las enseñanzas de disciplinas atléticas y gimnásticas pasaran a formar parte de un segundo plano en las clases de Educación Física. Un claro ejemplo es el caso del material autoconstruido, habiendo sido reportado que su uso puede resultar más útil, motivante y atractivo de cara al alumnado (Méndez-Giménez, Martínez de Ojeda-Pérez, \& Valverde-Pérez, 2016), ayudando a desarrollar no solo capacidades motoras sino también capacidades socioafectivas (García-Romero, 2016; Méndez-Giménez, Fernández-Río, \& Méndez-Alonso, 2015).

Al igual que se hace uso de material con fabricación no destinada a su uso en el aula de Educación Física, el material gimnástico y de atletismo disponible en los centros también podría ser utilizado para alcanzar otros objetivos no puramente gimnásticos o atléticos. Teniendo en cuenta que el profesorado encuestado ha sido de Educación Primaria, es comprensible que determinados materiales como el caballo con arcos, las paralelas o la cama elástica no sean utilizados para el desarrollo de habilidades altamente específicas de las habilidades gimnásticas. Sin embargo, sí que podrían ser materiales a utilizar con el objetivo de desarrollar otros aspectos del currículum escolar, dándole un uso más polivalente. En este punto, el profesorado encuestado también reporta que es un material de difícil manejo que podría incluso dañar el pavimento de la instalación. Los centros que poseen este tipo de material también deberían contar con los instrumentos necesarios para su transporte y colocación en el aula de forma segura e higiénica.

\section{Conclusiones}

La renovación de la enseñanza de la Educación Física, adaptada a pedagogía moderna e innovadora que busca cumplir objetivos de aprendizaje predeterminados, junto con el avance tecnológico que nos proporciona diversidad de materiales pedagógicos para utilizar en este ámbito, ha provocado que gran parte del material que hace tiempo era habitual en el aula de Educación Física quede relegado a un segundo plano o, en muchos casos, a caer en el desuso absoluto.

Es cierto que el material gimnástico y atlético propio de una Educación Física más cuantitativa y basada en objetivos de condición física ha quedado obsoleto y que los objetivos marcados en el currículum educativo actual se pueden lograr con el empleo de otro tipo de material más novedoso, atractivo, menos costoso o más seguro, por citar algunas características del material convencional actual que se emplea en Educación Física. Pero también es cierto que este tipo de material que ha quedado en el olvido puede proporcionar alternativas de uso ricas y creativas, siendo necesario buscar un uso polivalente y, de esta forma, aprovechar todos los recursos al alcance del docente, no pudiendo obviar que los datos proporcionados por este estudio nos dicen que la mayoría de este material está en buenas condiciones de conservación.

También se trata de buscar soluciones a los motivos por los que no se usa preferentemente este material. Problemas como la dificultad de movilidad de este material (en muchos casos pesado y voluminoso) o la peligrosidad (relativa) que puede provocar su uso en el aula (por su estructura, forma o tipo de material) se pueden solucionar con la adaptación de mecanismos de rodamiento o con el empleo de acolchados. En definitiva, se trata de buscar soluciones para usar todo tipo de materiales que tenemos a nuestra disposición para hacer una práctica más variada, rica y con multitud de posibilidades motrices para el alumnado. 


\section{Referencias}

Anderson, T., \& Kanuka, H. (2002). E-research: methods, strategies and issues. London: Allyn \& Bacon.

Amado, J. (2014). Manual de investigação cualitativa em educacão. Coimbra: Coimbra University Press.

Ávalos-Ramos, M. A., Martínez-Ruiz, M. A., \& Merma-Molina, G (2015). La pérdida de oportunidades del aprendizaje gimnástico: las voces del profesorado de Educación Secundaria. Ágora para la Educación Física y el Deporte, 17(2), 130-147.

Barcala-Furelos, R., Abelairas-Gómez, C., \& Gil-Madrona, P. (2016). Didáctica de la Educación Física en educación infantil (1. ${ }^{\mathrm{a}}$ ed.). Madrid: Síntesis.

Blández, J. (1995). La utilización del material y del espacio en Educación Física. Barcelona: INDE.

Buèar-Pajek, M., Èuk I., Kovaè M. \& Jakše, B. (2010). Implementation of the gymnastics curriculum in the third cycle of basic school in Slovenia. Science of Gymnastics Journal, 2(3), 15-27.

Cenizo-Benjumea, J. M., Seiva-Henares, P., \& Fernández-Truán, J. C. (2017). Autoeficacia del profesorado de Educación Física en la enseñanza de habilidades gimnásticas. EmásF. Revista Digital de Educación Física, 8(44), 41-55.

Delgado-Fernández, M., Valero-Valenzuela,A., \& Conde-Caveda, J. L. (2003). Justificación del atletismo como contenido curricular de primaria basándose en una propuesta práctica. Retos. Nuevas Tendencias en Educación Física, Deporte y Recreación, 5(1), 21-26.

Díaz-Lucea, J.(1996). Los recursos y materiales didacticos en educacion fisica. Apunts: Educacion Fisica y Deportes, 43(1), 42-52.

Fernádez-Río, J. F. (2010). Viejos y nuevos materiales y espacios en educación física: aprovechamiento y posibilidades. Tándem: Didáctica de la educación física, 32(1), 64-72.

Flick, U.(2015). El diseño de investigación Cualitativa. Madrid: Morata.

García-Romero, C.(2016). Percepción de los estudiantes de Educación Primaria sobre el material autoconstruido en la asignatura de Educación Física. Sportis. Revista Técnico-Científica del Deporte Escolar, Educación Física y Psicomotricidad, 2(2), 206-221.

Hardman, K. (2008). Physical education in schools: a global perspective. Kinesiology: International Journal of Fundamental and Applied Kinesiology, 40(1), 5-28.

Johnson, B., \& Onwuegbuzie, A. (2004). Mixed methods research: a research paradigm whose time has come. Educational Researcher, 33(7), 14.26.

Lima, L., \& Palhares, J. A. (2014). Metodología de investigação em Ciências Sociais da Educação. Braga: Humus.

López-Pastor, V. M., \& Gea-Fernández, J. M. (2010). Innovación, discurso y racionalidad en Educación Física. Revisión y prospectiva. Revista Internacional de Medicina y Ciencias de la Actividad Física y del Deporte, 10(38), 245-270.

Maeda, J. K., \& Burt, T. (2003). Inexpensive Equipment for Preschool Movement Activities. Teaching Elementary Physical Education, 14(2), 32.

Méndez-Giménez,A., Fernández-Río, J., \& Méndez-Alonso, D. (2012). Valoración de los adolescentes del uso de materiales autoconstruidos en educación física. Retos. Nuevas Perspectivas de Educación Física, Deporte y Recreación, 22(1), 24-28.

Méndez-Giménez,A., Martínez de Ojeda-Pérez, D., \& Valverde-Pérez, J. J. (2016). Valoración del alumnado y profesorado del material convencional y auto-construido: estudio longitudinal de diseño cruzado en Educación Deportiva Retos, Nuevas Tendencias en Educación Física, Deporte y Recreación, 30(1), 20-25.

Méndez-Giménez,A., Fernández-Río, J., \& Méndez-Alonso, D. (2015). Modelo de educación deportiva versus modelo tradicional: efectos en la motivación y deportividad. Revista Internacional de Medicina y Ciencias de la Actividad Física y el Deporte, 15(59), 449- 466.

Molina, J. P., Devís, J., \& Peiró, C. (2008). Materiales curriculares: clasificación y uso en educación física. Pixel-Bit. Revista de Medios y Educación, 33(3), 183-197.

Pereira, Z. (2011). Los diseños de método mixto en la investigación en educación: una experiencia concreta. Revista Electrónica Educare, 15(1), 15-29.

Rodríguez-Gómez, D. \& Valldeoriola, R. (2009). Metodología de la investigación. Barcelona: UOC.

Tabernero, B., \& Márquez, S. (2003). Estudio del Aula de Educación Física: Análisis de los Recursos Materiales Propios del Área. Apunts: Educación física y deportes, 72(2), 49-54.

Teixeira-Costa, H. J.,Abelairas-Gómez, C., Barcala-Furelos, R., \& ArufeGiráldez, V.(2015). The influence of a structured physical education plan on preschool children's psychomotor development profiles. Australasian Journal of Early Childhood, 40(2), 68-77.

Ugalde, N., \& Balbastre, F. (2013). Investigación cuantitativa e investigación cualitativa: buscando las ventajas de las diferentes metodologías de investigación. Revista de Ciencias Económicas, 31(2), 179-187.

United Nations Educational, Scientific and Cultural Organizations. (2015). Quality Physical Education. Guidelines for policy-makers. París: UNESCO.

Xunta de Galicia (2018). Consellería de Cultura, Educación e Ordenación Universitaria. Guía de centros educativos. Consulta e mantemento de centros. Recuperado de https://www.edu.xunta.es/ centroseducativos/BuscaCentros.do

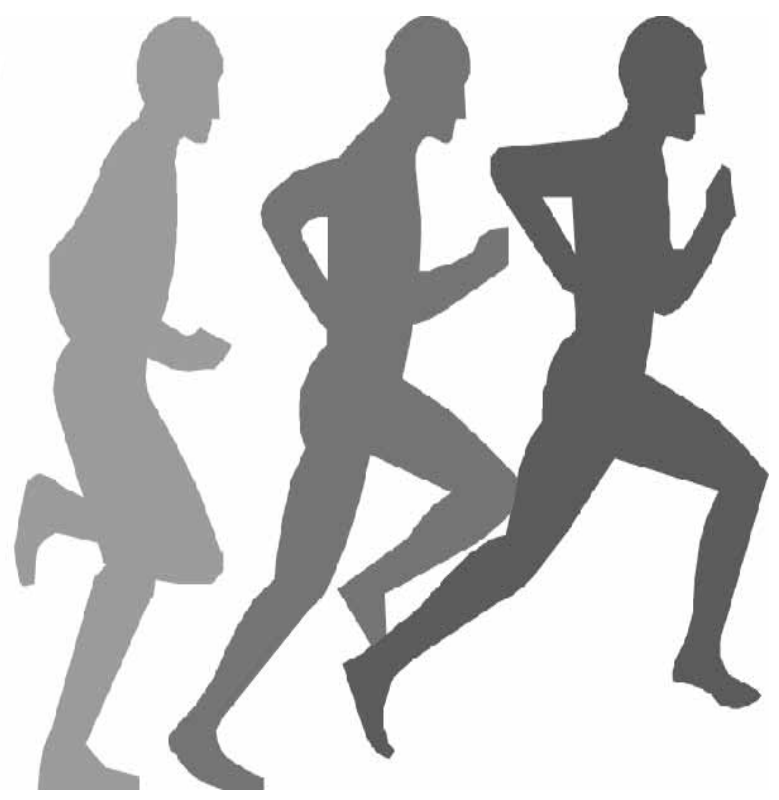

